\title{
Open issues in confinement, for the lattice and for center vortices
}

\section{John Cornwall*University of California at Los Angeles (UCLA)}

E-mail: cornwallephysics.ucla.edu

Center vortices have been around for more than thirty years, well-confirmed on the lattice, and very successful in explaining the basics of confinement, yet there are still open questions unstudied either on the lattice or in theory. The first is that basic confinement in the center vortex picture is topological, coming from gluonic solitons where the gluons have no direct coupling to the Wilson loop, and makes no reference to any particular surface (whose area would appear in the area law) or fluctuation dynamics of this surface. Only in $d=2$ (flat Wilson loops) is it obvious what surface must be involved, and in this dimension there is no room for fluctuations. This makes it hard to understand the Lüscher term and other properties of the fluctuating confinement surface for $d>2$. I make the obvious, but unconfirmed to date, conjecture that in topological confinement for non-planar Wilson loops the area law is the exponential of a string tension times the area of a minimal surface spanning the Wilson loop. Less obvious is whether, in this purely topological picture, this minimal surface shows the correct Lüscher term, or whether this term must come from gluons propagating between points on the Wilson loop (as possibly described by fishnet graphs and their relative the gluon chain model). Closely-related issues are the structure of the area law for two coaxial Wilson loops, as the distance between them along the axis grows; the resulting Casimir force between hadrons; and the behavior of $k$-string tensions for $S U(N)$ with $N>3$. I suggest a program of both lattice and theoretical studies, focused on center vortices and the pinch technique, to explore these and other issues: 1) Calculate the area law and its fluctuations for non-planar Wilson loops, or for pairs of flat Wilson loops, in a center-vortex-like ground state with a gas of vortices, but with no gluon-Wilson loop coupling. 2) Study more closely a picture I outline here of reconciling center vortices and minimal surfaces with fishnet graphs and the gluon-chain model, with the key ingredient of dynamically-massive gluons. 3) Extend beyond perturbation theory the old lattice work of Dashen and Gross on background-field Feynman gauge fixing to extract the gauge-invariant off-shell Green's functions of the pinch technique.

International Workshop on QCD Greens Functions, Confinement and Phenomenology September 7-11, 2009

ECT Trento, Italy

\footnotetext{
* Speaker.
} 


\section{Introduction}

Center vortices have been around so long [1] that one might think there is nothing new to say about them, and in fact most of the important developments, both in theory and on the lattice, are covered in Greensite's 2003 review article [2]. The general view among many of us at that time was that center vortices explained everything that needed explaining, although in certain cases the explanations might have been a bit skimpy. For example, center vortices explain the $\mathrm{N}$-ality dependence of confinement, including string breaking for $N$-ality zero representations; the lack of confinement in the exceptional Lie groups $G_{2}, F_{4}$, and $E_{8}$ that have trivial centers; along with nexuses (monopole-like objects) they generate non-integral topological charge; and are essential for chiral symmetry breakdown (CSB). But since 2003 many workers have turned to other issues; in particular, on the lattice it has become popular to study various gauge fixings, notably Landau and Coulomb gauges.

For center-vortex believers there are important questions still awaiting convincing answers, including the details of CSB and the $\theta$ dependence of quarkless $S U(N)$ gauge theories, but I will not discuss them here. Instead, I want to raise what seems to me to be an important point of principle so far not well-understood in QCD: What is a good first-principles explanation of the Lüscher term, given a confining condensate of center vortices? That is, how is a surface under tension formed spanning a Wilson loop? The reason this is not so well-understood is that it is easy to understand confinement as a purely topological phenomenon of the linking of center vortices with a Wilson loop, but the basic topology makes no reference to any spanning surface or its area. There are, of course, candidate non-topological mechanisms for forming a surface under tension that I (and Greensite, at this Workshop) will discuss, but it is also interesting to know whether or not a purely topological form of confinement through an ensemble of center vortices actually leads to a minimal surface with tension. This is a challenging problem only for non-planar Wilson loops, but it can be simulated much more easily than full QCD can be simulated.

Aside from purely topological generation of a Lüscher term, the other candidates are gluonchain models [Greensite, this Workshop; [3-5]] and fishnet graphs [6-8]. I argue that there is a kind of $d=4$ duality between the chromomagnetic, source-free gluons bound up in the closed two-surfaces of center-vortex solitons and gluons with chromoelectric sources that propagate from one point on a Wilson loop to another, while interacting with each other and forming a fishnet-like two-surface (or, at fixed time, a gluon chain). The chromoelectric gluons in the gluon chain are analogous to, or dual to, the localized monopole-like solitons $[9,10]$, which I call nexuses, that live on the center-vortex surface. Entropic effects coming from the coupling strength $\left(4 \pi / g^{2} \simeq 1\right)$ distinguish between electric and magnetic confinement. This kind of approximate duality only makes sense if the gluons have a dynamical mass $m$, which allows nexuses of mass $\sim 4 \pi \mathrm{m} / \mathrm{g}^{2}$ to exist.

The final point of this talk concerns the greatly-increased activity in lattice studies of off-shell Green's functions, where it has so far been necessary to fix to a particular gauge (usually Landau or Coulomb). This is hardly in the spirit of the original Wilson formulation of gauge theory, where one of the most important and basic features was gauge invariance. At this Workshop you will hear about recent advances in using the pinch technique [11-13], which is a way of constructing gauge-invariant off-shell Green's functions in the continuum. My colleagues Papavassiliou and 
Binosi, motivated by some one-loop calculations of others, proved [14] that to all orders the pinch technique is exactly the same as the background-field Feynman gauge. So my plea to the lattice workers here: Can you find a way of doing lattice simulations in this gauge? (Dashen and Gross [15] did it long ago to lowest order in the coupling, and Cucchieri et al. [16] have taken the first step toward an effective formulation of general $R(\xi)$ gauges, where $\xi=1$ is the Feynman gauge.)

\section{Center vortices}

The first question to ask is whether there is a convincing model of center vortices apart from the multiple lattice simulations (reviewed in [2]) showing that they exist. Just after center vortices were introduced by 't Hooft, I [17] pointed out that a dynamically-generated gluon mass made it easy to find center vortices as solitons ${ }^{1}$ of an infrared-effective action with a gauge-invariant mass term. The Schwinger-Dyson equations of the pinch technique $[13,18,19]$ showed that there was indeed a dynamical gluon mass of perhaps $600 \mathrm{MeV}$ driven by infrared slavery. This result has been repeatedly confirmed on the lattice (see the references in $[18,19]$ ). A dynamical gluon mass and the necessary long-range pure-gauge potentials are crucial ingredients in the picture presented here.

I want to connect the Lüscher term to (planar) fishnet graphs [6 -8]. Much of this early interest in fishnet graphs centered on the possibility of finding in them the Veneziano dual-resonance model. This is not my concern here, which is to find a description of a physical surface under tension. It will be critical that the gluons have a mass. Before going into the fishnet/gluon chain models, I will give a brief review of why the basic picture of center-vortex confinement is topological.

\section{Basic topological center-vortex confinement}

As far as confinement goes in QCD, there are two kinds of gluons. The first kind, type I (chromomagnetic), are the ones in the condensate of center vortices; these source-free gluons are parts of solitons that are only indirectly coupled to a Wilson loop. The second, type II (with chromoelectric sources such as quarks), are the ones that propagate from one point on a Wilson loop to another point, interacting with other gluons as they go. Correspondingly, there are two possibilities for forming a surface under tension, and I suspect that both of them play an important role. The first possibility is that, for deep mathematical reasons, the area in the area law for a condensate of random mutually-avoiding vortices formed from type I gluons is the area of a minimal surface and that this surface is under tension, for planar and non-planar Wilson loops. I suspect that this is so, and hope that the relatively straightforward lattice simulations (because type II gluons can be omitted) to investigate this question will soon be done. The second may lie in some approximation to the properties of fishnet graphs.

Let me briefly review the essential ingredients of topological confinement using a gaugeinvariant massive Abelian model, suppressing all irrelevancies. For simplicity of exposition I speak only of $d=3$ center vortices for a $U(1)$ subgroup of gauge group $S U(2)$, which are effectively Abelian closed $d=1$ loops, but everything goes through in $d=4$ where vortices are closed $d=2$

\footnotetext{
${ }^{1}$ In their simplest form, essentially the Abelian Nielsen-Olesen vortex of the Abelian Higgs model, but in fact much more complicated as we will discuss.
} 
surfaces. This model has long-range Goldstone-like fields that are necessary for gauge-invariant gluon masses. The action is of London form:

$$
I=\int d^{3} x\left\{\frac{1}{4} F_{i j}^{2}+\frac{m^{2}}{2}\left(A_{i}-\partial_{i} \phi\right)^{2}-i \oint_{\Gamma} d \tau \dot{z}_{i} \delta(x-z(\tau)) A_{i}\right\} ;
$$

the last term is the coupling to the current $J_{i}$ of the Wilson loop $\Gamma$.

Type I center vortices are Nielsen-Olesen-like solitons of this action minus the current term. The classical soliton is a sum (with given collective coordinates) of vortex terms of the form:

$$
A_{i}(x)= \pm \sum_{V} \pi \oint_{V} d z_{k} \varepsilon_{i j k} \partial_{j}\left[\Delta_{m}(x-z)-\Delta_{0}(x-z)\right] \equiv U_{i}
$$

$\Delta_{m, 0}$ is the free mass- $m$ or massless propagator, the sum is over a condensate of very long (compared to the persistence length; see below) closed loops labeled by $V$ that are mutually- and self-avoiding. The confining part of the solitonic gauge potential comes solely from the $\Delta_{0}$ term, which is a singular pure-gauge term coming from the Goldstone-like field $\phi$. (The short-distance Dirac-string singularities cancel between the two terms.) For purposes of studying the area law only the $\Delta_{0}$ term need be saved in Type I gluons.

In addition, there is a Type II term in the vector potential coming from gluons having the Wilson-loop current as source:

$$
\Delta_{m} J_{i} \equiv \int\left(\nabla^{2}+m^{2}\right)^{-1} J_{i}
$$

The relevant terms in the action that involve this loop are:

$$
I_{J}=\int\left\{-U_{i} J_{i}+\frac{1}{2} J_{i} \frac{1}{\nabla^{2}+m^{2}} J_{i}\right\}
$$

the first term in brackets is Type I, and the massive term is Type II. The Type II term is conventional and gives rise to a perimeter term. In our Abelian model the Type II gluons do not interact with each other, but it is essential for the Lüscher term that they do interact, as indeed they do in a non-Abelian gauge theory.

As the collective position coordinates of each loop $V$ vary, an ensemble of vortices is realized. For $S U(2)$ quarks, the VEV $\langle W\rangle$ of the Wilson loop $\Gamma$ is the ensemble average:

$$
\langle W\rangle=\left\langle\exp \left[i \oint_{\Gamma} d z_{i} A_{i}(z)\right]\right\rangle .
$$

Insert the $\Delta_{0}$ term of Eq. (3.2) to find:

$$
\langle W\rangle \equiv\left\langle\exp \left[i \pi \sum_{V} L k_{V}\right]\right\rangle
$$

where $L k_{V}$ is the Gauss link number of vortex $V$ with the Wilson loop, as given in the standard integral:

$$
L k_{V} \equiv \oint_{\Gamma} d x_{i} \oint_{V} d z_{k} \varepsilon_{i j k} \partial_{j} \Delta_{0}(x-z)
$$

Consequently, $\langle W\rangle$ is simply the ensemble average of a product of -1 's, with a -1 for every piercing by a vortex of any surface spanning the Wilson loop. 
This is an area law, as the well-known $d=2$ derivation shows. To implement a finite persistence length $\lambda$ put the vortices on a lattice of unit length $\lambda$, and the flat Wilson loop on a coordinate plane of the dual lattice. That portion of the coordinate plane bounded by the Wilson loop is a flat surface spanning the Wilson loop; it is pierced at places by vortices. To implement self-avoidance we take it that at most one vortex can pierce any unit square of this spanning surface, and we assign a probability $p$ that such a unit square is in fact pierced by a vortex. There is an areal density $\rho$ of vortex pierce points on the flat surface, equalling $p / \lambda^{2}$. Assume for simplicity that all vortex piercings are uncorrelated and that every piercing of a Wilson loop is equivalent to a contribution \pm 1 to the VEV (which is not true, but taking this into account only changes the value of, not the existence of, the string tension; see [20]). It is then easy to see that:

$$
\langle W\rangle=(\bar{p}-p)^{N}=\exp \left[\ln (1-2 p) A / \lambda^{2}\right] \equiv e^{-\sigma_{0} A}
$$

where $\bar{p}=1-p$ is the probability that a square of the spanning surface is not pierced by a vortex (thus giving a factor of 1 in the VEV) and $N=A / \lambda^{2}$ is the number of lattice squares in the spanning surface, of area $A$.

In this $d=2$ case it is pretty obvious what surface and what area is going to show up in the Wilson-loop VEV, but what about $d>2$ and non-planar Wilson loops? It is true that by Stokes' theorem the Gauss link number can be displayed as an integral showing the piercing by a vortex of some surface spanning the Wilson loop, but any surface will do, since the link number is purely topological. Yet the law of confinement is expressed solely in terms of the Wilson loop contour and must make reference to a (generically) unique surface for a given contour. One can see semiquantitatively [20] that the larger the area of a spanning surface the fewer vortices are actually linked (the rest have, for $S U(2)$, an even Gauss link number), which is to say that the probability $p$ of Eq. (3.8) depends on the surface and diminishes as the surface area grows. Maybe then it is plausible that the area to be associated with linked vortices, in the sense of Eq. (3.8), is a minimal area. But to my knowledge this has never been shown convincingly from first principles.

Since only Type I gluons contribute to the area law, it is much simpler to simulate the area laws for non-planar Wilson loops because one need not simulate the full dynamics of QCD. All that is needed is to construct an ensemble of random, self- and mutually-avoiding closed loops and to calculate the area law and its fluctuations from topological formulas such as Eq. (3.6). Construction of such ensembles is well-known in polymer physics [see, for example, [21]]. It would be very interesting to prove or disprove the conjecture that purely topological confinement leads to a minimal surface and Lüscher term. Equally fascinating is the study of area laws for more complex Wilson loops, such as the change in the area law with $z$ of two coaxial loops separated by a distance $z$ [20].

\section{Fishnet graphs and gluon chains}

There is no convincing first-principles explanation that I know telling us why the area in the area law is like a physical membrane with a surface tension and not just a mathematical area somehow defined by the topology of a sea of vortices. (Of course, there is a lot of lattice evidence and no one doubts the reality of this surface tension.) I will make some remarks that probably are no more convincing than any other hand-waving explanation, but which I feel are on the right 


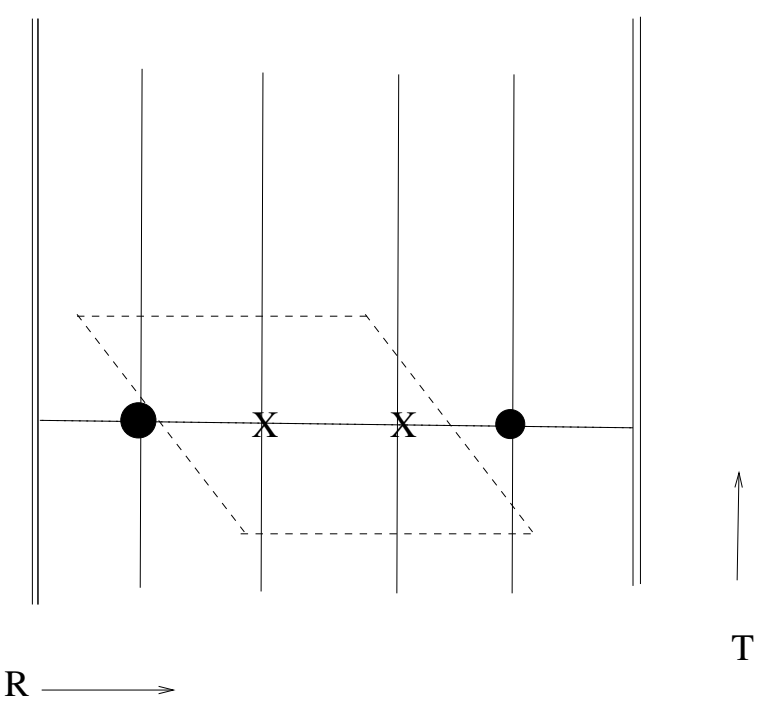

Figure 1: Double lines are the edges of one Wilson loop. Dashed lines show a perpendicular Wilson loop. Dotted lines with circles and Xs show gluon chain in R direction, intersecting the dashed-line Wilson loop at the Xs.

track. They have to do with the old subject of fishnet graphs [6-8], and the less old subject of gluon chains [3-5]. One of the motivating factors for gluon chains is that they lead to a picture rather like that of string in string theory, and should naturally accommodate a Lüscher force and related phenomena.

Begin with gluon chains. In a simplistic description of previous work, a gluon chain joining a static quark and antiquark (which I think of as two opposite sides of a large Wilson loop) consists of a string of localized gluons joined to their nearest neighbors (including quarks) by a force that increases no less rapidly than linear. I show a sketch of the chain in Figure 1. In the figure the gluons are shown as localized to a particular time, but of course they actually have world lines (the vertical lines in the figure). The long string of gluon nearest-neighbor interactions, at fixed time, reduces this growth to linear (or if the original gluon-gluon force was linear, may reduce the string tension, as needed in Coulomb gauge [5]). Since the gluons are supposed to be massless, some sort of unspecified localization mechanism has to exist. Moreover, it is not clear what exactly gives the linear or super-linear force law between nearest-neighbor gluons in the first place.

There is a kind of dual to the gluon-chain model in which gluons are replaced by nexuses ${ }^{2}$ (essentially chromomagnetic monopoles) joined by chromomagnetic flux tubes, whose energy naturally grows linearly with separation $[9,10,22]$. If a gluon has dynamical mass $m$, a nexus has mass of order $4 \pi m / g^{2}$, where $g^{2} /(4 \pi) \sim \mathscr{O}(1)$ is the QCD running coupling in the infrared. In fact, a center vortex in $d=3(4)$ is a closed string (two-surface) carrying the chromomagnetic flux of the nexuses, which, as point-like (world-line) objects, divide the string (surface) into domains of differing flux orientations. There are also center vortices, with a single orientation, with no

\footnotetext{
${ }^{2}$ Nexuses are strictly non-Abelian and cannot exist in isolation, but only as parts of center vortices; when nexuses are included, center vortices are much more complex objects than the simple Nielsen-Olesen vortex.
} 
nexuses. ${ }^{3}$ In $d=4$ the closed center-vortex two-surface can terminate on an 't Hooft loop. 'T Hooft's confinement criterion [1] says that if there is electric confinement (an area law for the Wilson loop) there is no confinement for the 't Hooft loop. The way it comes about is that electric confinement requires a dominant entropy contribution to the center-vortex action, in which case the center vortices, or their constituents the nexus chains, tend to be very long and space-filling. In such a case the magnetic "quarks" in the 't Hooft loop feel no long-range force, just as if two of them were joined by a floppy string very long compared to the quarks' separation. Conversely, there is no entropy-driven $d=3,4$ condensate of ordinary gluons in QCD.

Another statement of this duality is to think of the gluon chains as a $d=2$ "condensate", which needs a source - a Wilson loop. This condensate can also be described as a fishnet graph, with massive gluons. The mass provides a localization mechanism for the gluons, which we can idealize as non-relativistic. So the mass is also the dominant source of string energy. In the (purely hypothetical) limit of chromoelectric-magnetic duality, the properties of the $d=2$ gluon-chain condensate should be much like the properties of a $d=2$ slice of the space-filling vortex condensate living in $d=3,4$. I have already reduced this vortex condensate to $d=2$ terms in the discussion of topological confinement, with an areal density $\rho_{M}=p / \lambda^{2}$ (the subscript $M$ indicating that the condensate is of chromomagnetic vortices). Equate this to the intergluon density $\rho_{E}$ of the gluon chain model, where $\rho_{E} \approx \zeta^{2}$, in terms of the intergluon spacing $\zeta$. The gluons have mass $m$, so the energy of a gluon chain of length $R$ is

$$
\varepsilon=\sigma R \approx m \zeta R
$$

Earlier I argued that $\sigma \approx 2 \zeta^{2}$, so $\zeta \approx m / 2, \sigma \approx m^{2} / 2$. This happens to work fairly well for $m \approx$ $600 \mathrm{MeV}$.

Next, the question of the Lüscher term, which I discuss in very sketchy terms. Figure 2 shows a Wilson loop decomposed as a fishnet graph; for simplicity, I deal only with four-gluon interaction terms explicitly and treat gluons as massive scalar particles. Of course, the fishnet graph is not this regular lattice, since all vertex coordinates must be integrated over. I assume that the average distance between four-gluon vertices is of the order of $\lambda$, the intervortex distance introduced earlier. Let $\sigma_{1}$ be a coordinate in the plane along the $R$ direction, and $\sigma_{2}$ a coordinate in the $T$ direction. Every four-gluon vertex has $d=4$ coordinates $Z_{\beta}\left(\sigma_{1}, \sigma_{2}\right)$. As in [7] write the value $G$ of the fishnet graph as:

$$
\begin{aligned}
G & =\text { const. } \times \prod_{\sigma}\left\{d^{4} Z_{\beta}(\sigma) \Delta\left[(x-y)\left(\sigma_{a}\right)\right]\right\} \\
& =\text { const } . \times \int\left(d^{4} Z_{\beta}\right) \exp \left\{\sum_{\sigma} \ln \Delta\left[(x-y)\left(\sigma_{a}\right)\right]\right\}
\end{aligned}
$$

Approximate the propagation distances $x-y$ by the first term in a derivative expansion, so that with:

$$
\ln \Delta\left[(x-y)^{2}\right]=\int_{0}^{\infty} \frac{d s}{s} \int_{x}^{y}(d z) \exp \left\{-\frac{m}{2} \int_{0}^{s} d \tau\left[\dot{z}^{2}+1\right]\right\}
$$

\footnotetext{
${ }^{3}$ The linkage of ordinary center vortices with nexus world lines is one way of producing non-integral topological charge [23-25].
} 


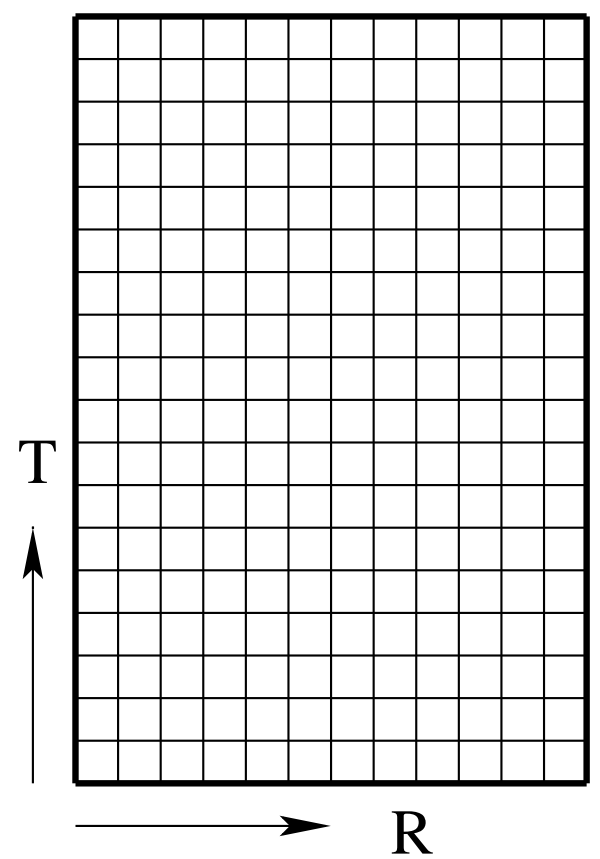

Figure 2: A fishnet graph in a Wilson loop (heavy lines). Vertices labeled by coordinates $Z_{\beta}\left(\sigma_{1}, \sigma_{2}\right)$; lines point along unit vectors $\hat{e}(R, T)$

(dots indicate proper-time derivatives) and the expansion:

$$
\begin{aligned}
x_{\beta} & =Z_{\beta}\left(\sigma_{a}+\lambda \hat{e}_{a}^{R, T}\right), y_{\beta}=Z_{\beta}\left(\sigma_{a}\right) \\
x_{\beta}-y_{\beta} & \approx \lambda \hat{e}(R, T) \cdot \partial Z_{\beta}(\sigma)
\end{aligned}
$$

(where, for example, $\hat{e}(R)$ is a unit vector in the $R$ direction). I further assume that the proper-time integral over $s$ can be replaced by multiplication by $\Delta s \approx \lambda \approx 1 / m$. Sum over unit vectors to find the fishnet graph value roughly:

$$
G \approx \int\left(d Z_{\beta}\right) \exp \left\{-\int \frac{d^{2} \sigma}{\lambda^{2}} \frac{m}{2 \Delta s}\left[\left(\lambda \partial_{a} Z_{\beta}\right)^{2}\right]\right\}
$$

The semiclassical approximation to $G$ yields the equation $\left(\partial_{a}\right)^{2} Z_{\beta}=0$. We interpret this as coming from a force-balance relation at every vertex, with the gluon forces along the world lines meeting at the vertices. If two world lines are in the $R$ direction and the other two in the $T$ direction this force balance reads:

$$
\begin{aligned}
Z_{\beta}\left(\sigma_{1}+\lambda, \sigma_{2}\right)-Z_{\beta}\left(\sigma_{1}, \sigma_{2}\right) & +Z_{\beta}\left(\sigma_{1}-\lambda, \sigma_{2}\right)-Z_{\beta}\left(\sigma_{1}, \sigma_{2}\right) \\
& + \text { two other terms }=0 \rightarrow \lambda^{2}\left(\partial_{a}\right)^{2} Z_{\beta}=0 .
\end{aligned}
$$

However, this specification is incomplete, since the world lines need not point along the coordinate axes and they can join anywhere. The physical situation is something like what is shown in Figure 


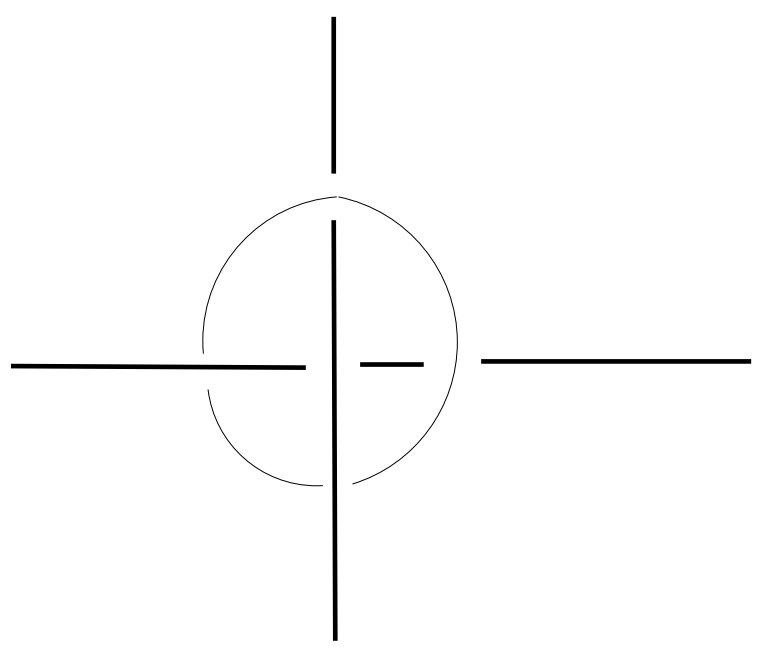

Figure 3: Heuristics of vertex equilibria: Like two strings held together by a frictionless ring. They can slide freely, but they can't separate

3, showing two frictionless strings bound at one point by a frictionless ring that allows sliding but not separation of the strings. A further specification of force balance is:

$$
\partial_{a} Z_{\beta} \partial_{b} Z_{\beta}=0(a \neq b)
$$

and isotropy demands:

$$
\partial_{1} Z_{\beta} \partial_{1} Z_{\beta}=\partial_{2} Z_{\beta} \partial_{2} Z_{\beta}\left[=f^{2}(\sigma)\right]
$$

In other words the induced metric is in conformal gauge:

$$
\eta_{a b}=\partial_{a} Z_{\beta} \partial_{b} Z_{\beta}=\delta_{a b} f^{2}(\sigma)
$$

Now there are many minima of the fishnet action of Eq. (4.2) if no further conditions are imposed, but if the conformal gauge condition is imposed, the action is the Dirichlet action of a minimal surface. The force-balance conditions used here support the obvious conjecture that a fishnet graph indeed corresponds to a surface under tension, with as usual a Lüscher term.

\section{A return to gauge invariance on the lattice?}

There is by now a vast body of lattice simulations done in the last few years in particular gauges, notably the Landau gauge. This seems to violate the basic premise of lattice gauge theory, which is gauge invariance; no gauge-fixing terms were needed to define on-shell functional integrals. The apparent price to pay for gauge invariance was that we were forbidden to look at off-shell Green's functions. And if a gauge is fixed, allowing off-shell calculations, the Green's functions were not gauge-invariant and hence unphysical. Nevertheless there is good reason for lattice theorists and simulators to do gauge-fixed simulations, because otherwise the toolset for understanding the mechanisms of QCD is too limited. Only with off-shell Green's functions can one 
construct and solve Schwinger-Dyson equations (SDEs), to understand, for example, how gluons get a dynamical mass and many other issues.

In the continuum we have the pinch technique (PT) [11 - 13] that showed how to extract offshell Green's functions that really were gauge-invariant, at least at the one-loop level. In a general gauge this requires a complicated recombination of standard Feynman graphs and graph pieces. Years later, Papavassiliou and Binosi (who will tell you more about it in this Workshop) made the ultimate advance, showing (after some suggestive one-loop work by others) that the all-order generalization of the PT was nothing but good old Feynman graphs calculated in the backgroundfield Feynman gauge [14]; no recombination necessary in this gauge.

It is perhaps ironic that the way to find PT Green's functions is to choose a particular gaugethe background-field Feynman gauge. The point is, of course, not that a priori specification of this gauge is particularly important, but that the gauge-invariant off-shell Green's functions of the PT happen to be those calculated in this gauge.

There are at least two ways to do the PT rearrangement on the lattice.

1. Translate into lattice language the steps used in the continuum PT, in which parts of standard Green's functions in any gauge, for example Landau gauge, are recombined, using Ward identities and other special tools, and added together in gauge-invariant combinations.

2. Make use of the work of Dashen and Gross [15] and of Cucchieri et al. [[16] and this Workshop] to find out how to implement the background-field Feynman gauge in lattice simulations.

Neither of these approaches is easy, or they would be done by now.

The first way is very complex, but may be useful because it can start from the Landau gauge, which is by now well-understood on the lattice. As for the second way, Dashen and Gross [15] long ago showed how to implement gauge-fixing to the background-field Feynman gauge on the lattice, but only in lowest-order perturbation theory. Cucchieri and collaborators [see [16] and references therein] spent considerable effort trying to generalize the usual Landau gauge-fixing algorithm, which is in fact a minimization over gauge transformations, to general covariant gauges as specified by the standard $\xi$-parameter $(\xi=0$, Landau gauge; $\xi=1$, Feynman gauge). But for $\xi \neq 0$ there is no simple minimization procedure that generalizes Landau gauge. Cucchieri et al. have recently found [16] a new formulation of $R(\xi)$ gauges that, while not trivial to implement on the lattice, is more promising for numerical implementation than earlier efforts. Since Cucchieri is talking at this Workshop on this subject I will not discuss it further here, but just comment that to me, at least, there seems no particular extra difficulty in principle to extend the work in [16] to the background-field Feynman gauge, using the work of Dashen and Gross.

I urge the community of lattice theorists and simulators to think about this: How can you construct gauge-invariant off-shell Green's functions for the lattice, and calculate their properties? 


\section{References}

[1] G. 't Hooft, On the phase transition towards permanent quark confinement, Nucl. Phys. B 138 (1978) 1.

[2] J. Greensite, The confinement problem in lattice gauge theory, Prog. Part. Nucl. Phys. 51 (2003) 1 [arXiv:hep-lat/0301023].

[3] G. Tiktopoulos, Gluon Chains, Phys. Lett. B 66 (1977) 271.

[4] J. Greensite and C. B. Thorn, Gluon chain model of the confining force, JHEP 0202 (2002) 014 [arXiv:hep-ph/0112326].

[5] J. Greensite and S. Olejnik, Constituent gluon content of the static quark-antiquark state in Coulomb gauge, Phys. Rev. D 79 (2009) 114501 [arXiv:0901.0199 [hep-lat]].

[6] H. B. Nielsen and P. Olesen, A parton view on dual amplitudes, Phys. Lett. B 32 (1970) 203.

[7] B. Sakita and M. A. Virasoro, Dynamical model of dual amplitudes, Phys. Rev. Lett. 24 (1970) 1146.

[8] G. 't Hooft, A planar diagram theory for strong interaction, Nucl. Phys. B 72 (1974) 461.

[9] J. Ambjorn and J. Greensite, Center disorder in the 3D Georgi-Glashow model, JHEP 9805 (1998) 004 [arXiv:hep-lat/9804022].

[10] J. M. Cornwall, Nexus solitons in the center vortex picture of QCD, Phys. Rev. D 58 (1998) 105028 [arXiv:hep-th/9806007].

[11] J. M. Cornwall, Confinement and infrared properties of Yang-Mills theories, invited talk at the US-Japan Seminar on Geometric Models of the Elementary Particles, Osaka, June 1976 (available on SPIRES).

[12] J. M. Cornwall, Nonperturbative mass gap in continuum $Q C D$, invited talk at the French-American Seminar on Theoretical Aspects of Quantum Chromodynamics, Marseille, France, 1981 (Centre de Physique Théorique report CPT-81/P-1345, ed. J. W. Dash, Marseille, 1982).

[13] J. M. Cornwall, Dynamical mass generation in continuum QCD, Phys. Rev. D 26 (1982) 1453.

[14] D. Binosi and J. Papavassiliou, The pinch technique to all orders, Phys. Rev. D 66 (2002) 111901 [arXiv:hep-ph/0208189]; Pinch technique self-energies and vertices to all orders in perturbation theory, J. Phys. G30 (2004) 203 [arXiv:hep-ph/0301096].

[15] R. F. Dashen and D. J. Gross, The relationship between lattice and continuum definitions of the gauge theory coupling, Phys. Rev. D 23 (1981) 2340.

[16] A. Cucchieri, T. Mendes and E. M. S. Santos, Covariant gauge on the lattice: a new implementation, [arXiv:0907.4138 [hep-lat]].

[17] J. M. Cornwall, Quark confinement and vortices in massive gauge invariant QCD, Nucl. Phys. B 157 (1979) 392.

[18] D. Binosi and J. Papavassiliou, New Schwinger-Dyson equations for non-Abelian gauge theories, JHEP 0811 (2008) 063 [arXiv:0805.3994 [hep-ph]].

[19] A. C. Aguilar, D. Binosi and J. Papavassiliou, Gluon and ghost propagators in the Landau gauge: Deriving lattice results from Schwinger-Dyson equations, Phys. Rev. D 78 (2008) 025010 [arXiv:0802.1870 [hep-ph]].

[20] J. M. Cornwall, Probing the center-vortex area law in $d=3$ : The role of inert vortices, Phys. Rev. D 73 (2006) 065004 [arXiv:hep-th/0601090]. 
[21] T. Vettorel, A. Y. Grosberg, and K. Kremer, Statistics of polymer rings in the melt: a numerical simulation study, Physical Biology 6 (2009) 25013.

[22] J. M. Cornwall, Center vortices, nexuses, and the Georgi-Glashow model, Phys. Rev. D 59 (1999) 125015 [arXiv:hep-th/9901039].

[23] J. M. Cornwall, Dynamical problems of baryogenesis, in Unified Symmetry: In the Small and in the Large, ed. B. N. Kursonoglu et al, p. 243, Plenum Press, New York, 1995.

[24] M. Engelhardt and H. Reinhardt, Center projection vortices in continuum Yang-Mills theory, Nucl. Phys. B 567 (2000) 249 [arXiv:hep-th/9907139].

[25] J. M. Cornwall, Center vortices, nexuses, and fractional topological charge, Phys. Rev. D 61 (2000) 085012 [arXiv:hep-th/9911125]. 